\title{
Evaluation of Peripheral Lower Limb Insufficiency among Patients with Diabetes Mellitus using Doppler Ultrasound - An Prospective Study
}

\author{
S. Fareed UI Hameed ${ }^{1}$, S. Habeeb Mohammed ${ }^{2}$, P. Karuppasamy ${ }^{3}$ \\ ${ }^{1}$ Assistant Professor, Department of General Surgery, ${ }^{2}$ Professor \& HOD, Department of General Surgery, ${ }^{3}$ Associate Professor, \\ Department of General Surgery, Melmaruvathur Adhiparasakthi Institute of Medical Sciences and Research, Melmaruvathur, \\ Kancheepuram, District Tamilnadu, India
}

Corresponding author: Dr. S. Habeeb Mohammed, Professor \& HOD, Department of General Surgery, Melmaruvathur Adhiparasakthi Institute of Medical Sciences and Research, Melmaruvathur, Kancheepuram, District Tamilnadu, India

DOI: http://dx.doi.org/10.21276/ijcmsr.2020.5.1.1

(c) BY-NC-ND

How to cite this article: S. Fareed UI Hameed, S. Habeeb Mohammed, P. Karuppasamy. Evaluation of peripheral lower limb insufficiency among patients with diabetes mellitus using doppler ultrasound - an prospective study. International Journal of Contemporary Medicine Surgery and Radiology. 2020;5(1):A1-A5.

\section{A B S T R A C T}

Introduction: Diabetes mellitus is a common pathological condition of the present times, with a higher prevalence rate in developing countries. It has a marked impact on the quality of life due to the acute and long-term complications secondary to the involvement of micro and macro vasculature with the progression of the natural history of disease. The Doppler ultrasound is non-invasive and can be used as a means of evaluating vascular complications in diabetics. Hence, the aim of the present study was to determine the frequency, level and flow patterns in lower limb arterial insufficiency among diabetic patients with the help of Doppler ultrasound study.

Materials and Methods: The present prospective study was done in December 2011 to December 2012 on 100 patients admitted in the Krishna institute of medical sciences, Karad. Adult diabetic patients (aged 18 years or above) irrespective of type of diabetes and gender with suspected peripheral vascular insufficiency or complaints of numbness, discoloration of periphery or ulceration were taken in this study.

Results: The most common artery involvement was found to be dorsapaedalis artery in $26 \%$ of the cases followed by proximal femoral and distal femoral in $18 \%$ of the cases whereas there were $12 \%$ cases with popliteal artery and distal artery branches involvement. The most common site of lesion involved was proximal femoral artery and dorsapaedalis artery seen in $26 \%$ of the cases (both) whereas distal femoral artery (DFA) was found in $10 \%$ of the cases, poplitial artery (PA) in $18 \%$ of the cases followed by posterior tibial artery (PTA) and anterior tibial artery (ATA) in $8 \%$ and $12 \%$ of the cases respectively.

Conclusion: Peripheral vascular insufficiency was a significant finding in patients having diabetes for 1-10 years. The dorsalispaedis artery was the commonest site of involvement. The normal Doppler study virtually rules out a haemodynamically significant lesion and helps to prevent unnecessary intervention.

Keywords: Doppler Ultrasound, Diabetes Mellitus, Peripheral Arterial Disease, Lower Limb

\section{INTRODUCTION}

Diabetes is a worldwide problem. A majority of diabetes patients develop foot ulcers at one point of time or other during the course of their illness. A significant number of such patients will require long-term hospital treatment and amputations. In the first half of this century, foot lesions in diabetics, which frequently lead to amputation, were considered an unavoidable complication of diabetes associated with arteriosclerosis and were classed under the heading of "diabetic gangrene. It was not until the 1950s that diabetic neuropathy, ischemia and infection, were finally recognised as precondition of foot complications in diabetics- fact that still hold good today. ${ }^{1,2}$

Peripheral Artery Disease (PAD) is a contributing factor to the development of foot ulcers in up to $50 \%$ cases and there is increased risk of amputations and hospitalisation with increasing severity of infection. It commonly affects the tibial and peroneal arteries of the calf. Ultrasound Colour Doppler method is valuable, non-invasive tool that may provide good information about the anatomy and physiology of the vessels. Computed Tomography Angiography (CTA) provides higher resolution and can scan the entire volume quickly. It helps in knowing the exact level and extent of arterial blockage but is costly. ${ }^{3}$

Diabetic foot is a long-standing diabetes complication in which infection, ulceration, and/or necrosis of tissues of the lower limb occurs, owing to presence of neuropathy and peripheral artery disease as basic etiological factors. The foot ulcer incidence rate ranges between $2 \%$ and $10 \%$ among patients with diabetes mellitus. According to various studies, there is 15-46 fold higher Lower Limb Amputation 
risk (LLA) in diabetics as compared to general population. Furthermore the diabetic foot expense consumes about 15\% of overall hospital budget for diabetes. ${ }^{4}$

Colour Doppler ultrasound is a widely available non-invasive technique in the assessment of arterial affection of lower limbs that occur with DM. Colour Doppler ultrasound has lots of advantages over other diagnostic imaging modalities; it is a feasible modality available in nearly all hospitals, being cheap, accurate, and safe. It also uses nonionizing radiation, and hence is safe in all cases with no limitations. ${ }^{5}$

This colour Doppler ultrasound technique is not used with contrast agents, and therefore it has no hazards of allergic or nephropathic effects and it competes with magnetic resonance angiography, and some studies showed same or better results compared with magnetic resonance angiography but with much less cost. ${ }^{6}$

It can identify the condition of arterial wall, whether healthy or diseased, atheromatous plaques and calcification through gray scale. Areas of stenosis, turbulence, and defect of blood flow can be seen with the help of colour. Moreover, a major advantage for Doppler ultrasound over other imaging modalities is that it can identify hemodynamic changes that occur with arterial stenosis. ${ }^{7,8}$

Diagnosis of PAD in diabetic patients is of great clinical significance in order to determine the subset of patients that may be at a high risk of subsequent myocardial infarction or stroke regardless of the presence or absence of symptoms of PAD and to treat the symptoms of PAD, which can lead to limb loss and functional disability. Thus, regular screening is essential as a part of aggressive management to reduce the impact of comorbidities on the affected person. ${ }^{9}$ Hence, the aim of the present study was to determine the frequency, level and flow patterns in lower limb arterial insufficiency among diabetic patients with the help of Doppler ultrasound study.

\section{MATERIAL AND METHODS}

The present study was a prospective study which was conducted in December 2011 to December 2012, among the patients admitted in Krishna Institute of Medical Sciences, Karad. Total 100 patients were included in this study by purposive non-randomized sampling. Adult diabetic patients (aged 18 years or above) irrespective of type of diabetes and gender with suspected peripheral vascular insufficiency or complaints of numbness, discoloration of periphery or ulceration were included in this study. Patients with a previous history of trauma to the arterial vasculature, those suffering from other causes of peripheral arterial insufficiency like hypertension, vitamin deficiency, and known atherosclerotic disease, and those who underwent arterial graft procedures were excluded.

Demographic data was collected to analyse details such as identification, gender, age, address, presenting complaints, treatment history and level of $\mathrm{Hb} \mathrm{A} 1 \mathrm{c}$ (when available). Doppler ultrasound (U/S) was done by a convex linear array probe of $11.5 \mathrm{MHz}$ on Toshiba Nemio-17. Colour and spectral Doppler technique were used to find out the affected vessel. Mapping of the lower limb arterial tree was performed, from common iliac up to the dorsalispaedis artery.

Presence of significant ischemia was confirmed with the measurement of ankle to brachial index ratio (ABI), involving the measurement of peak systolic flow velocities in the ankles (dorsalis paedis and posterior tibial arteries), and arms (brachial artery) by using a handheld Doppler and then calculating a ratio, with an $\mathrm{ABI}$ of $0.9-1.3$ indicating normal flow, 0.6-0.9 indicating mild ischemia, 0.4-0.6 indicating moderate ischemia and $<0.4$ severe ischemia.

Ultrasonic Doppler equipment is for detecting and evaluating blood flow. In ultrasonography, transducers convert electrical energy into mechanical energy to produce ultrasound and vice versa. Five types of diagnostic Doppler instruments are usually distinguished such as (1) Continuous Wave (CW) Doppler (2) Pulsed Wave (Pw) Doppler (3) Colour Doppler Imaging (Cdi, Colour Velocity Imaging) (4) Duplex Doppler (5) Power Doppler Imaging.

\section{STATISTICAL ANALYSIS}

The data was entered into the Microsoft excel sheet and was analysed with the help of SPSS software version 21. Descriptive statistics was performed and was presented in the form of tables and graphs.

\section{RESULTS}

In the present study, out of 100 cases, $68 \%$ were males and $32 \%$ were females (Graph 1). The majority of cases belonged to 51-60 years followed by 61-70 years (Graph 2). The patients with Type I diabetes mellitus was found to be $40 \%$ followed by Type II diabetes in $60 \%$ of the cases (Graph 3). The newly detected diabetes cases were found to be $35 \%$ whereas the majority of cases with diabetes since 1-10 years were found to be $38 \%$ (Graph 4). The vascular abnormality

\begin{tabular}{|c|c|c|}
\hline Arterial Doppler & No. of patients & Percentage \\
\hline Normal & 14 & $14 \%$ \\
\hline Generalized atherosclerosis narrowing only & 55 & $55 \%$ \\
\hline Generalized atherosclerosis narrowing only+ arterial block only & 31 & $31 \%$ \\
\hline Total & 100 & $100 \%$ \\
\hline
\end{tabular}

\begin{tabular}{|l|c|c|}
\hline Arterial block in Doppler & Amputation & Percentage \\
\hline Present & 21 & $80.1 \%$ \\
\hline Absent & 5 & $19.2 \%$ \\
\hline Total & 26 & $100 \%$ \\
\hline \multicolumn{2}{|c|}{ Table-2: Shows the distribution of data according to arterial Doppler and amputation among the study subjects } \\
\hline
\end{tabular}




\begin{tabular}{|l|c|c|}
\hline Artery involve in stenosis & Number of cases & Percentage \\
\hline Dorsapaedalis alone & 26 & $18 \%$ \\
\hline Proximal femoral and Distal femoral & 18 & $12 \%$ \\
\hline Popliteal artery and distal arterial branches & 12 & $12 \%$ \\
\hline Posterior tibial with dorsapaedalis artery & 12 & $10 \%$ \\
\hline Posterior tibial artery alone & 10 & $12 \%$ \\
\hline Popliteal artery alone & 12 & $10 \%$ \\
\hline Common ilieac and distal arterial branches & 10 & \\
\hline \multicolumn{2}{|c|}{ Table-3: Shows the distribution of data according to arterial involvement in stenosis among the study subjects } \\
\hline
\end{tabular}

\begin{tabular}{|l|c|c|}
\hline Level & Number of cases & Percentage \\
\hline PFA & 26 & $26 \%$ \\
\hline DFA & 10 & $10 \%$ \\
\hline PA & 18 & $18 \%$ \\
\hline PTA & 8 & $8 \%$ \\
\hline ATA & 12 & $12 \%$ \\
\hline DPA & 26 & $26 \%$ \\
\hline
\end{tabular}

Table-4: shows the distribution of data based on site of lesion by Doppler ultrasound among the study subjects

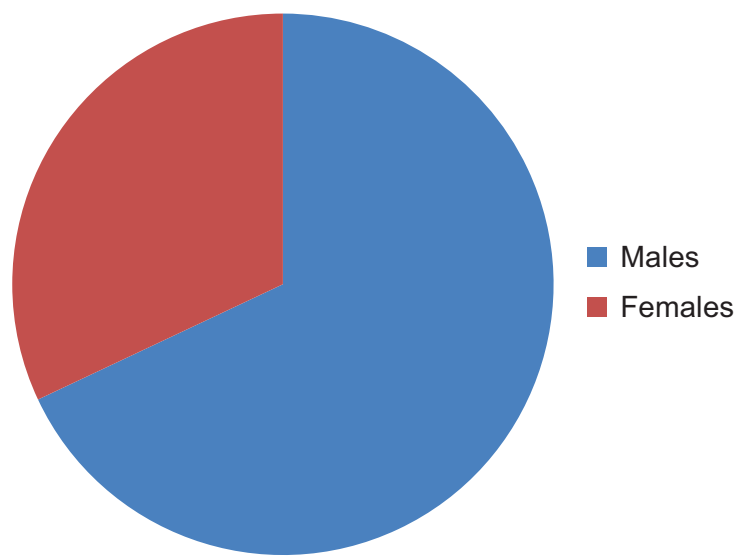

Graph-1: Shows the distribution of data according to gender among the study subjects

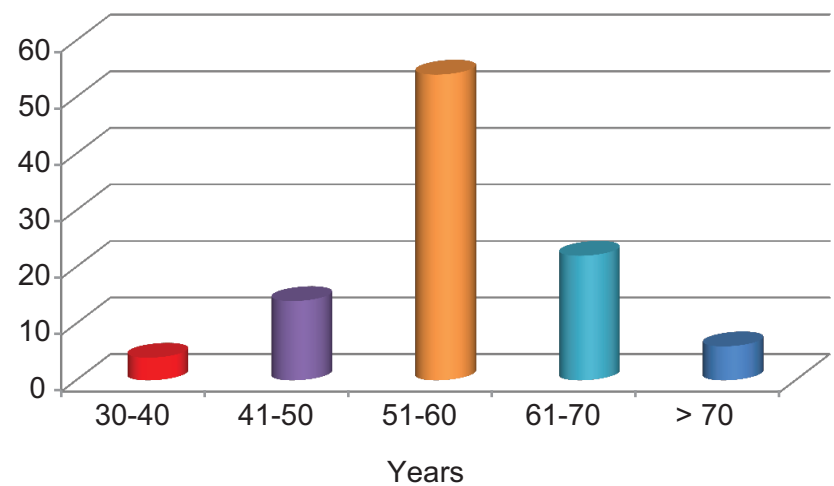

Graph-2: Shows the distribution of data based on age group among the study subjects

was found to be $72 \%$ and normal cases were found to be $28 \%$ in this study (Graph 5). The involvement of right limb was $48.8 \%$ and left limb was found to be $32.5 \%$ followed by bilateral involvement in $18.6 \%$ cases (Graph 6). Majority of the cases showed generalized atherosclerosis narrowing in 55\% of the cases whereas generalized atherosclerosis narrowing only plus arterial block was found in 31\% of

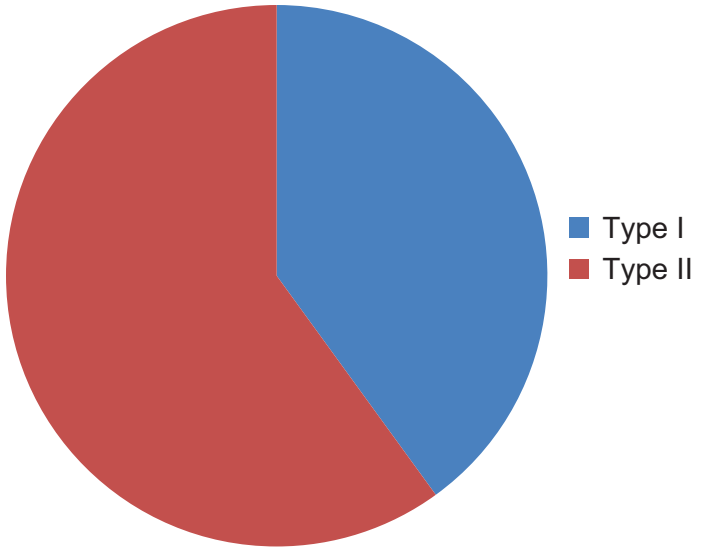

Graph-3: Shows the distribution of data according to type of diabetes mellitus among the study subjects

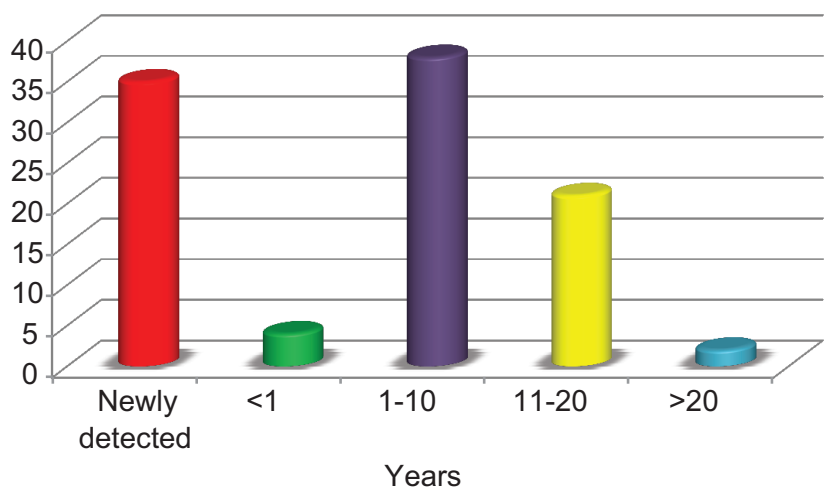

Graph-4: Shows the distribution of data based on duration of diabetic status among the study subjects

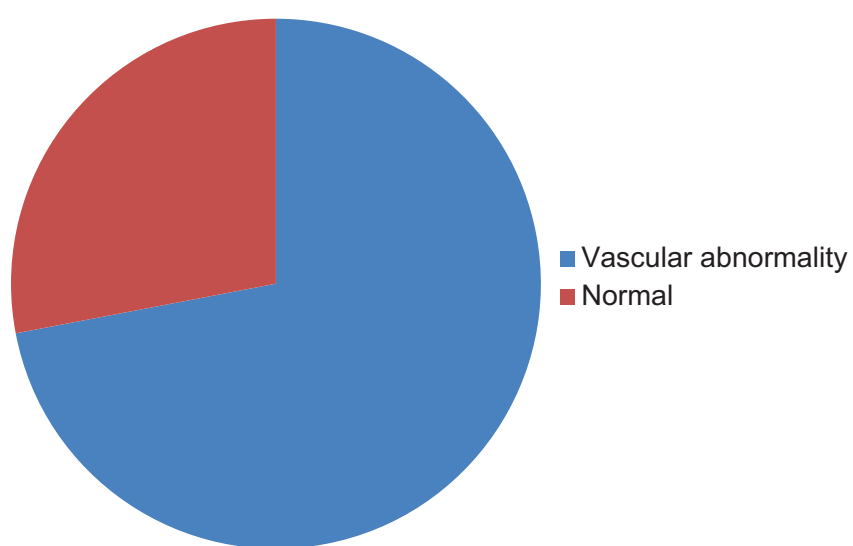

Graph-5: Shows the distribution of data based on Doppler evidence in limbs among the study subjects

the cases (Table 1). It was observed that about $80.1 \%$ were present with amputation and about 19.2\% did not 


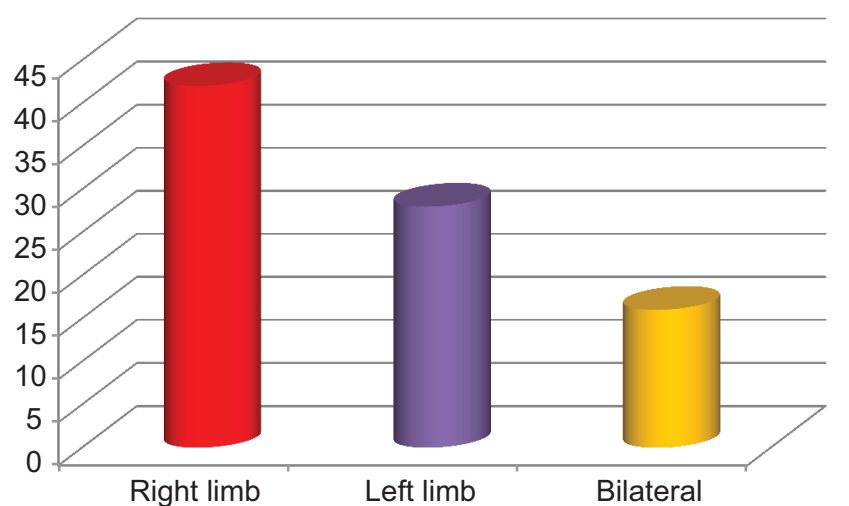

Graph-6: Shows the distribution of data based on side of limb involvement among the study subjects

showed any amputation (Table 2). The most common artery involvement was found to be dorsapaedalis artery in $26 \%$ of the cases followed by proximal femoral and distal femoral in $18 \%$ of the cases whereas there were $14 \%$ cases with popliteal artery and distal artery branches involvement (Table 3). The most common site of lesion involved were proximal femoral artery (PFA) and dorsapaedalis artery seen in $26 \%$ of the cases (both) whereas distal femoral artery (DFA) was found in $10 \%$ of the cases, popliteal artery (PA) in $18 \%$ of the cases followed by posterior tibial artery (PTA) and anterior tibial artery (ATA) in $8 \%$ and $12 \%$ of the cases respectively (Table 4).

\section{DISCUSSION}

The concept of diabetic foot syndrome incorporates various clinical pictures characterized by different etiologies and pathological mechanisms. Common to all is the fact that injuries to the foot of diabetic patient can results in complication that may lead to amputation of the limb if treatment is delayed or ineffective. Diabetic foot lesions are common in middle aged person i.e. in $4^{\text {th }}$ and $5^{\text {th }}$ decade of life and this may be attributed to many factors like duration of diabetes, history of addiction to smoking or alcohol and hyperlipidaemia along with hyperglycaemia which together accelerates the process of plaque formation in arteries of lower extremities. Male predominance was also encountered in a previous similar study by Das et al which reported that out of 60 patients examined for diabetic lower limbs arterial disease, $60 \%$ were male and $40 \%$ were female. ${ }^{10,11}$

Higher incidence of diabetic foot lesion is found in males is attributed to smoking, trauma, and unhygienic habits. As males are the breadwinners of the family and are mostly working out door, which makes them more vulnerable for trauma and sequelae. Most of the patients were having diabetes of long duration, have higher chances of developing foot lesions. Most of the patients presented with history of some kind of injury to the foot before the onset of the lesion, because in long standing diabetes there is neuropathy which results in loss of sensation and the patient will be unaware of the injury and neglects it. ${ }^{12}$

This is in agreement with previous studies by Guo et al., who found higher results than the present study as regards bilateral and multi-segmental diabetic lower limbs arterial affection. About 53\% of patients examined were affected by bilateral lower limb arterial disease and 70\% had multisegmental distribution. ${ }^{13}$

In a study conducted by Zeinab Ali et al reported that diabetic lower limb arterial disease had more affection of the distal i.e. below knee arteries in about $66 \%$ of patients. The DPA was the most commonly involved artery with significant stenosis in our study (26\%); the femoral artery was the second most commonly affected at $18 \%$. There were similar results of arterial stenotic characteristics and segmental distribution of diabetic lower limb arterial disease in previous studies. In a study made by Das et al, the ATA (in 31\%) and PTA (in 30\%) were the most commonly involved arteries with luminal narrowing and these results are not in concordance with the findings of the present study. ${ }^{14,11}$

The development of atherosclerosis in the course of diabetes consists in the process of vessel wall remodeling. At the early stage of atherosclerosis, remodeling allows to maintain the flow lumen as it consists in centrifugal thickening of vessel wall with increase in overall vessel diameter (outward remodeling). In a more advanced stage of atherosclerosis, the wall undergoes centripetal hypertrophy, leading to stenosis of the flow lumen (inward remodeling). Pathophysiology of vessel remodeling was first described by Galgov. The type and size of remodeling depends on the production of vascular epithelial growth factor, proteases and cellular adhesion molecules as a response to the changes in blood flow. ${ }^{15,16}$

The amputation rate is much lower as compared to other studies, due to better education of the patient, better glycaemic control, and proper care of foot followed by proper usage of antibiotics and extensive debridement and regular dressing. Hyperlipidaemia in diabetic foot patients accelerate the process of formation of atheromatous plaque in intima media of the arteries. Thus, along with good glycaemic control hyperlipidaemia should also be under control to minimise diabetic foot complication and hazards of amputations. ${ }^{17,18}$

\section{CONCLUSION}

Middle aged person are at increased risk of peripheral arterial atherosclerotic disease associated with diabetes. Duplex sonography can accurately locate the site and extent of stenosis/ occlusion with advantages of low cost, non-invasive in nature, outpatient procedure within less time. Doppler ultrasound being a non-invasive is an easily repeatable procedure.

\section{REFERENCES}

1. Sheikh MZ. Diabetes mellitus: the continuing challenge. J Coll Physicians Surg Pak 2004; 14(1):63-4.

2. Martinez-Castelao A. Clinical and social impact of the diabetes mellitus epidemic. Nefrología 2008; 28(5):2458.

3. Marso SP, Hiatt WR. Peripheral arterial disease in patients with diabetes. J Am Coll Cardiol 2006; 47(2:921-9.

4. Hittel N, Donnelly R. Treating peripheral arterial disease in patients with diabetes. Diabetes Obes Metabol 2002; 4(6):S26-31.

5. Jude EB, Oyibo SO, Chalmers N, Boulton AJ. Peripheral arterial disease in diabetic and non-diabetic: 
a comparison of severity and outcome. Diabetes Care 2001; 24(1):1433-7.

6. Koelemay MJ, Legemate DA, de Vos H, van Gurp AJ, Balm R, Reekers JA, et al. Duplex scanning allows selective use of arteriography in the management of patients with severe lower limb arterial disease. J Vasc Surg 2001; 34(3):661-7.

7. American Diabetes Association. Peripheral arterial disease in people with diabetes. Diabetes Care 2003; 26(6):3333-41.

8. Zimmet P, Aberti KG, Shaw J. Global and societal implications of the diabetes epidemic. Nature 2001; 414(6865): 782-87.

9. Wild S, Roglic G, Green A et al. Global Prevalence of Diabetes: Estimates for the year 2000 and projections for 2030. Diabetes Care 2004; 27(5): 1047-53.

10. Bosevski M. Peripheral arterial disease and diabetes. Prilozi 2012; 33(1):65-78.

11. Das G, Gupta AKr, Aggarwal A. Assessment of lower limb arteries by Doppler sonography in diabetic patients. Int J Res Health Sci 2015; 3(4):18-23.

12. Chidambara PK, Swaminathan RK, Ganesan $P$, Mayavan M. Segmental comparison of peripheral arteries by Doppler ultrasound and CT angiography. J Clin Diagn Res 2016; 10(3):TC12-TC16.

13. Guo XJ, Shi YX, Huang XZ, Ye M, Xue GH, Zhang JW. Features analysis of lower extremity arterial lesions in 162 diabetes patients. Diabetes Res Clin Pract 2013; 2013:781360.

14. Zeinab A. Ali Rehab M. Habib, Mahmoud A. M. Abo Hendy. Comparative study between Doppler ultrasound and computed tomography angiography in diabetic lower limb arterial insufficiency. Menoufia Med J 2018; 31(2):1050-1057.

15. Rahman S, Rahman T, Ismail AAS, Rashid ARA. Diabetes-associated macrovasculopathy: pathophysiology and pathogenesis. Diabetes Obes Metab 2007; 9(5):767-780.

16. He C, Yang J-G, Li Y-M, Rong J, Du F-Z, Yang Z-G, et al. Comparison of lower extremity atherosclerosis in diabetic and non-diabetic patients using multidetector computed tomography. BMC Cardiovasc Disord 2014; 14(3):125.

17. Kandasamy G, Maithrayee A, Kailasanathan N. Lower limb arteries assessed with Doppler angiography. A prospective comparative study with multi detector CT angiography. Int J Latest Res Sci Tech 2015; 4(3):70-83.

18. Suzuki E, Egawa K, Nishio Y, Maegawa H, Tsuchiya M, Haneda M, et al. Prevalence and major risk factors of reduced flow volume in lower extremities with normal ankle-brachial index in Japanese patients with type-2 diabetes. Diabetes Care 2003; 26(7):1764-9.

Source of Support: Nil; Conflict of Interest: None

Submitted: 05-09-2019; Accepted: 15-11-2019; Published online: 03-12-2019 